\title{
MULTIFOCAL INDEPENDENT SPIKE SYNDROME
}

The relationship of the syndrome of multifocal independent spikes (MIS) to hypsarrhythmia and the slow spike-wave (Lennox-Gastaut) syndrome was studied in 64 children with MIS examined during a 3-year period at the Cleveland Clinic, Ohio. Fifteen additional patients had hypsarrhythmia, 17 had generalized slow spike-wave complexes (SSWC), and 22 had MIS and SSWC in the same recording. Transitions occurred from one pattern to another in 25/40 patients with 2 or more serial EEGs at least 5 months apart. All 25 patients showed the following transition sequence: Hypsarrhthmia --> MIS -- > MIS and generalized spikes --> SSWC. None of 8 patients with SSWC showed transitions, showing that SSWC is a stable pattern.

In another group of 20 patients with MIS, hypsarrhythmia, and SSWC examined prospectively over a 6 month period, sleep activated additional spike foci, increased the frequency of generalized spike discharges, and produced synchronization of bitemporal and bifrontal spike-wave discharges at 1.5-2.5 $\mathrm{Hz}$, the same as SSWC. The MIS pattern lies between hypsarrhythmia and SSWC ontogenetically; it is unstable and evolves to other EEG patterns. It should be regarded as a distinct syndrome, not as a variant of the Lennox-Gastaut syndrome. (Kotagal P. Multifocal independent spike syndrome: Relationship to hypsarrhythmia and the slow spike-wave (Lennox-Gastaut) syndrome. Clin Electroencephalogr January 1995;26:23-29). (Reprints: Prakash Kotagal MD, Section of Pediatric Epilepsy, Desk S-51, Cleveland Clinic Foundation, 9500 Euclid Avenue, Cleveland, $\mathrm{OH} 44195)$.

COMMENT. The multifocal independent spikes EEG record is defined as three or more independent foci of spikes or sharp waves occurring in multiple locations in both hemispheres. The voltage of the background activity does not show the high voltage of hypsarrhythmia (ie. it is less than $200 \mathrm{mcV}$ ). Well developed slow spike-wave complexes are absent, but occasional generalized spike discharges occur. The author believes that children with MIS syndrome and those with Lennox-Gastaut should not be lumped together. In the slow spike-wave syndrome (LennoxGastaut), generalized slow spike and slow-wave complexes at $1.5-2.5 \mathrm{~Hz}$ occur in a burst of three or more spike-waves in a row. The SSWC are the dominant discharges, but multifocal spikes may be seen in the same record. The Cleveland Clinic finds the MIS syndrome to be 3-4 times as common as the Lennox Gastaut syndrome and a distinct type of symptomatic generalized epilepsy in childhood.

\section{ASYMMETRIC HYPSARRHYTHMIA}

The clinical, EEG, and radiological findings in 6 patients with the asymmetric variant of hypsarrhythmia among 26 children with infantile spasms are reported from the University of Michigan EEG Laboratory, Ann Arbor, MI. The spasms were symptomatic of cerebral dysplasia in 4, porencephaly in 1 , and hypoxic-ischemic encephalopathy in 1 . Focal abnormalities on neurologic exam or imaging study were found in 5 children. The abnormal EEG activity was ipsilateral to the lesion in 4 and contralateral in 1. Of hypsarrhythmia EEGs seen in this lab, $23 \%$ were asymmetric. The EEG may show focal abnormalities that are not detected by clinical exam or imaging study. (Drury I, Beydoun A, Garofalo EA, Henry TR. Asymmetric hypsarrhythmia: Clinical electroencephalographic and radiological findings. Epilepsia Jan 1995;36:41-47). (Reprints: Dr I Drury, EEG Laboratory, University 
Hospital 1B300/0036, 1500 E Medical Center Dr, Ann Arbor, MI 48109).

COMMENT. The EEG may identify patients with infantile spasms whose lesions are amenable to surgery, even when not detected by clinical findings and imaging studies. Most cases showing asymmetric hypsarrhythmia had developmental abnormalities of the brain, primarily large cystic lesions. Cerebral tumors have been reported, eg. Branch CE and Dyken PR. Choroid plexus papilloma and infantile spasms. Ann Neurol 1979;5:302. The spasms resolved after surgery for removal of the tumor. These cases favor a primary cortical rather than brainstem origin for infantile spasms.

A generalized electrodecremental EEG resulting from a focal cortical ictal discharge and associated with partial seizures is reported in 23 patients seen at the Johns Hopkins Epilepsy Center (Arroyo S et al. Epilepsia 1994;35:974).

The surgical treatment of an early epileptic encephalopathy with suppression-bursts (Ohtahara's syndrome) and focal cortical dysplasia is reported from Hopital Pellegrin, Bordeaux, France (Pedespan JM et al. Epilepsia Jan 1995;36:37-40). Infantile spasms and brief left-sided tonic unilateral seizures began at 5 days of age. The interictal EEG showed an asymmetrical suppression-burst pattern affecting the right hemisphere. MRI showed right frontotemporal cortical thickening. Seizures were resistant to AEDs and steroid therapy. The right precentral area resected showed cytoarchitectural dysplasia and ectopic neurons deep in subcortical white matter. At 1 year follow-up, the child had suffered only a "febrile seizure," and had minor developmental delay with slight left-sided weakness. Surgery may be effective in some cases of Ohtahara's syndrome.

Sarnat HB, University of Washington, Seattle, reviews "Ependymal reactions to injury" and focal dysplasias of the developing brain that may be secondary to damage to fetal ependyma. (I Neuropathol Exp Neurol January 1995;54:1-15).

\section{EPISODIC HYPERSOMNOLENCE EPILEPSY}

A 4 year old child who had complex partial seizures alternating with sleep that presented as episodic hypersomnolence is reported from the Mayo Clinic, Rochester, MN. The hypersomnolence lasting 24-72 hrs was preceded by irritability and hyperkinesia for 48-96 hrs. The intervals between episodes ranged from 10-60 days. The EEG showed left occipital spikes in sleep. Prolonged recording during hypersomnolence showed right temporal seizure discharges. Phenytoin controlled the episodic hypersomnolence, with no recurrence at $19 \mathrm{yr}$ follow-up. Complex partial seizures followed by postictal somnolence occurred occasionally between 5 and $11 \mathrm{yrs}$ of age. CT was normal. Behavioral problems required special education and methylphenidate. He graduated from high school and has a manual labor job. (Wszolek ZK, Groover RV, Klass DW. Seizures presenting as episodic hypersomnolence. Epilepsia Jan 1995;36:108-110). (Reprints: Dr DW Klass, Section of Electroencephalography, Mayo Clinic, 200 First St SW, Rochester, MN 55905).

COMMENT. The EEG recording showing right temporal seizure discharges during the period of hypersomnolence. and the excellent response to phenytoin suggest that the episodic sleep disorder may be regarded as a seizure phenomenon. Lennox WG refers to "sleeplike episodes" and 